\title{
UN ANALISIS DIFERENCIAL DE LA FECUNDIDAD
}

Inmaculada Santos del Campo

Una de las mayores dificultades, en el estudio teórico y metodológico de la fecundidad, es la complejidad de este fenómeno. La determinación de la fecundidad está, tanto relacionada con factores biológicos como psicológicos, sociológicos o económicos. Es por todo esto que su estudio requiere un enfoque interdisciplinario en el cual tienen que integrarse diferentes niveles de análisis.

Sin embargo, muchos autores han intentado ponerlo en relación únicamente con una variable explicativa.

Así, por ejemplo, desde el punto de vista biológico, existen diferentes teorías basadas tanto en la diferenciación entre fecundidad (comportamiento reproductivo) y fertilidad (capacidad fisiológica), como en la relación entre desarrollo económico y fecundidad, caso de Josué de Castro, para el cual el nexo de unión entre ambos conceptos, desarrollo económico y fecundidad, es la dieta.

La idea generalizada entre los demógrafos es que el descenso de la fecundidad se ha producido de una forma voluntaria. Es decir, mediante prácticas de control y limitación de la fecundidad, ya sea mediante anticonceptivos o incluso el aborto. 
Sin embargo y a pesar de que esto hoy en día pueda ser más o menos cierto, no debemos olvidar que el deseo de limitar la familia -el número de hijos- ha sido una ilusión constante durante todo el desarrollo de la humanidad, es decir, en la mayoría de las sociedades preindustriales, y, sin embargo, éstas, ya sea por lo precario de los métodos conocidos, ya sea por la propia estructura social, han tenido un mayor número de hijos que en la actualidad.

\section{I}

¿Cuáles son, pues, los factores que pueden permitirnos explicar los diferentes comportamientos que se dan en materia de fecundidad?

Si bien, y como decía anteriormente, el fenómeno en sí no se puede estudiar, sino desde la múltiple interconexión de las variables que lo afectan como tal fenómeno, es esta misma complejidad la que, en definitiva, nos lleva a estudiar la fecundidad como una variable «dependiente», sometida por tanto a múltiples influencias. El buen conocimiento de estas influencias y de su delimitación teórica, será el único camino que nos permitirá hacer inteligible el fenómeno fecundidad.

Así pues, y con base en las proposiciones clásicas de Kingsley Davis, Judith Blake y Ronald Freedman, podría asumirse que la fecundidad depende básicamente de los siguientes factores que la condicionan:
a) La formación y permanencia de las uniones sexuales relativamente establecidas;
b) La orientación de la conducta reproductiva hacia un número de hijos
grande, mediano o pequeño (significación económica de los hijos); y
c) El uso de medios para prevenir la actividad sexual y sus consecuen- cias reproductivas (medidas anticonceptivas en el sentido amplio) o eliminar estas consecuencias (aborto).

Así pues, si partimos de que la razón más importante en los diferenciales de fecundidad, es el factor o factores de tipo socio-económico, tendremos que, en una primera aproximación, el lugar de residencia: urbano o rural, situará las condiciones que favorecerán o no el aumento o descenso de la fecundidad. Pero, eso sí, siempre teniendo en cuenta que este factor (lugar de residencia) está a su vez fuertemente interrelacionado con otros como movilidad social, nivel cultural, ocio... Dentro de estas variables explicativas las podemos desglosar en:

- Tipo de trabajo o situación laboral de la mujer. 
- Categoría socio-profesional del marido.

- Ingresos familiares.

- Nivel de vida.

- Problemas de vivienda.

- Instrucción del marido y de la mujer.

- Religión.

- Edad en el momento de contraer matrimonio...

Así pues, como podemos observar, la fecundidad no es una variable independiente; su incremento o su descenso estará vinculado a otros incrementos o descensos, y en especial a los de la economía.

\section{I I}

Sin embargo, la concreción del problema a nivel de España, nos lleva a un planteamiento de la cuestión en unos términos más cotidianos, en una primera aproximación para intentar confirmarla posteriormente con una serie de datos técnicos o estadísticos.

A unos términos más cotidianos en cuanto que debemos enmarcarlo no sólo en esta época y país concreto, sino también en la magnitud sociológica que el fenómeno comporta en la actualidad.

Efectivamente, uno de los grandes contrastes de este siglo en que vivimos es el crecimiento explosivo de la población en grandes partes del mundo, mientras que, simultáneamente, se registra en otras partes un fuerte descenso de la natalidad. Estas tendencias son dos efectos de la misma civilización, aunque bajo aspectos totalmente diferentes.

Los avances de la medicina y de la higiene moderna han supuesto un importante progreso en la lucha contra las enfermedades. De tal forma que se han creado las condiciones necesarias para conseguir un fuerte descenso de la mortalidad en cualquier parte del mundo. Por otra parte, estos mismos avances científicos han supuesto, frente a la natalidad incontrolada, una serie de instrumentos que han permitido adaptar la natalidad a los deseos de los padres, nacidos en una nueva sociedad industrial moderna, $y$, sobre todo, a las mujeres, que en esta sociedad cumplen un papel muy diferente. La mujer tiene en esta sociedad la posibilidad y la necesidad de trabajar fuera de casa.

Y la cuestión así planteada me lleva a asegurar que esta "posibilidadnecesidad» de trabajar fuera de casa está extrañamente e indisolublemente ligada a la renuncia a la maternidad.

Es un poco escribir contracorriente, escribir de un tema tan en desuso y decadente, como actualmente y sobre todo en países en vías de desarrollo, como es España, supone la maternidad. Porque, frente a este concepto se sitúa otro mucho más «moderno» y mucho más en «progreso»: el «feminis- 
mo». $\mathrm{Y}$ he puesto todos estos conceptos entrecomillados porque, efectivamente, creo que han perdido todo su significado. Mientras a cambio los hemos ido llenando de unas connotaciones tan políticas, tan manipuladas, que merecen un análisis aparte.

Efectivamente, la mujer, hoy en día, tras luchas más o menos duras, ha conquistado una serie de derechos que indudablemente le pertenecían, pero que de la misma forma sigue sin ejercer. Es decir, la mujer ha conseguido más o menos el derecho a estudiar y a trabajar en casi todos los campos. pero aun así esto resulta en la práctica muy incierto, la selección es rigurosa, desde la educación primaria, o en la educación familiar, hasta esa educación social latente en la vida cotidiana de los medios de información. Pero, en fin, las mujeres, sólo muy pocas mujeres acceden a la enseñanza superior; las otras, una gran, enorme mayoría, trabajan hasta la edad de casarse, e incluso hasta él o los primeros hijos, pero trabajan principalmente en el sector servicios, en sus oficinas, bancos, comercios, cafeterías, administración en general, etc. Para la mujer este trabajo que la libera, que la induce a creerse una persona independiente, no supone lo mismo que para el hombre. Sabe perfectamente que prefiere trabajar fuera de casa, que en el hogar; no en sí por el trabajo, sino más bien por la interacción social que su trabajo la supone.

¿Pero qué significa para una mujer con un trabajo no cualificado, de tipo medio, la maternidad? Pues algo terriblemente oneroso, ya que en el mejor de los casos la aislará de su vida social una vez terminado su trabajo diario, y en el peor, la obligará a abandonar ese trabajo que, sin embargo, la ha constituido como ente social. Ese terrible, paulatino e inexorable descenso de la fecundidad que se produce en todos los países en desarrollo y desarrollados, por la incorporación de la mujer a la actividad laboral, es una de las máximas conquistas, que no se deben al movimiento feminista, como éste suele creer, sino simplemente a la propia dinámica de nuestro sistema de producción.

Sin embargo, este esperado y ansiado descenso de la fecundidad, para una gran mayoría de los países, acaba, para otros, convirtiéndose en su peor pesadilla. $Y$ es que es difícil mostrar a una mujer lo que supone la vida social del trabajo y después negársela, e incluso, en este caso, que suele producirse en las recesiones económicas, la mujer ya ha aprendido una serie de técnicas anticonceptivas, pero sobre todo y mucho más importante ha aprendido que el concepto maternidad ha dejado de ser un concepto «eterno», para pasar a ser un concepto moldeable y manejable.

El problema, pues, se centra en que, hoy en día, se están creando una serie de «futuras» madres que aborrecen la maternidad, la aborrecen absolutamente, en cuanto que este concepto se ajusta perfectamente al de esclava, al de mujer de su hogar, a familia... al de formar otra vez parte de esa subclase social de la mujer. $\mathrm{Y}$ así se aferran, frente a esos hijos «no deseados», 
a un trabajo tampoco deseado, pero que les reporta más satisfacciones sociales que las incomodidades y sacrificios que requiere la maternidad.

Sí, es evidente que la fecundidad está descendiendo en España y también nos parece evidente que seguirá descendiendo en un país donde la incorporación de la mujer al trabajo es todavía muy pequeña (20 por 100 de la población total femenina y 27 por 100 de la población total activa).

Ha pasado ya a la historia la hora de las clases pasivas; hoy una pareja, para vivir normalmente y medianamente bien, necesitan trabajar los dos. En esta interacción la maternidad, en la mayoría de los casos, es un accidente molesto que hace descender el nivel de renta, el nivel de ocio, la movilidad espacial...

El sistema, pues, tiene sus propias contradicciones y éstas se manifiestan de mil formas diferentes, pero desde luego una de las más importantes es esa mal llamada "planificación demográfica», a la que también nos somete. Porque el tener un hijo, salvo en dudosas ocasiones, no responde a deseos. sino a las posibilidades reales de tenerlo.

Siempre se ha dicho que no es lo mismo un hijo que nace en el medio rural que en el medio urbano (aunque hoy en día empieza a presentarse también como bastante dudoso), en aquél tiene un claro valor: trabajará, ayudará en el trabajo familiar, su coste se verá, pues, superado por su valor en trabajo. En cambio, en el medio urbano, el hijo ya ha dejado de ser un valor deseable, porque es un coste y no solamente económico, sino también social y cultural. Se podría decir que, a mayor número de hijos, menos oportunidades económicas, sociales y culturales tiene una pareja. De tal forma que no es nada raro que en los países occidentales, con sus determinadas pautas de progreso, la fecundidad más alta se encuentre entre la clase alta y la clase más baja. Ambas, por diferentes motivos, no tienen nada que perder con esa mayor fecundidad, y, en cambio, ganan con el placer de su descendencia. Es, pues, esta gran, amplia masa media, que compone día a día nuestros países occidentales; esa gran cląse media, que se resiste a descender en el escalón social y consumista; que cree y espera una movilidad social soñada. Es esta clase media la que se niega a tirar sus ilusiones por la ventana, la que, en definitiva, hace descender esa famosa tasa de fecundidad.

\section{V}

Ya para terminar y como colofón gráfico de lo anteriormente expuesto, me veo en la necesidad de dar una serie de datos numéricos que colaboren a ilustrar este paulatino declinar de la fecundidad en España.

Una medida resumen de la fecundidad de una población es la tasa global de fecundidad (TGF). Representa el número de hijos que tendría una mujer 
que termine su período fértil, si a lo largo de ese período estuviera sujeta a las tasas de fecundidad por edad del intervalo de tiempo considerado (generalmente un año). Otro índice bastante utilizado es la tasa bruta de natalidad, que representa el número de nacimientos anuales que se producen por cada mil habitantes de la población en estudio. A pesar de su sencillez, esta medida tiene la desventaja de que está afectada por la estructura por sexo y edad de la población, efecto inexistente en la TGF.

Los datos disponibles más recientes muestran que España tenía en 1975 una tasa de natalidad de 19 nacimientos por 1.000 habitantes y una tasa global de fecundidad de 80,4 por 1.000 hijos (por cada mil mujeres). Estos índices no son tan bajos como las de la mayoría de los países desarrollados, pero sí, como podemos observar, empiezan a estar muy próximos.

En el cuadro 1 se compara la fecundidad y la natalidad de algunos países seleccionados (de forma absolutamente arbitraria) de Europa a mediados de la década de los sesenta y principios de la década de los setenta. Es ésta una situación nueva, pues recién empezado el siglo éramos todavía un país subdesarrollado en nuestras pautas de fecundidad (también en las de mortalidad). En cambio, se ha producido gracias a un paulatino descenso a lo largo de todo el siglo, como se puede apreciar en el cuadro 2, y si en un principio se puede achacar tanto al impacto de la guerra europea, como a la depresión económica posterior y a la guerra civil, es posteriormente a los años cincuenta una cuestión diferente con características propias, que señalan ya a otro orden de cosas.

\section{CUADRO 1}

Tasas de natalidad y tasas globales de fecundidad en países seleccionados de Europa, 1965, 1970 y 1972

\begin{tabular}{|c|c|c|c|c|}
\hline \multirow{2}{*}{ Países } & \multirow{2}{*}{$\begin{array}{l}\begin{array}{l}\text { Tasa global de } \\
\text { fecundidad }(\%)\end{array} \\
1965\end{array}$} & \multicolumn{3}{|c|}{$\begin{array}{l}\text { Tasa bruta de } \\
\text { natalidad }(\%)\end{array}$} \\
\hline & & 1965 & 1970 & 1972 \\
\hline $\begin{array}{cccccccccc}\text { Francia } & \ldots & \ldots & \ldots & \ldots & \ldots & \ldots & \ldots & \ldots & \ldots\end{array}$ & 28,3 & 17,8 & 16,8 & 17,0 \\
\hline $\begin{array}{llllllllll}\text { Grecia } & \ldots & \ldots & \ldots & \ldots & \ldots & \ldots & \ldots & \ldots & \ldots\end{array}$ & 68,3 & - & - & 16,1 \\
\hline $\begin{array}{llllllllll}\text { Italia } & \ldots & \ldots & \ldots & \ldots & \ldots & \ldots & \ldots & \ldots & \ldots\end{array}$ & 75,6 & 19,1 & 16,9 & 16,4 \\
\hline $\begin{array}{llllllllll}\text { Holanda } & \ldots & \ldots & \ldots & \ldots & \ldots & \ldots & \ldots & \ldots & \ldots\end{array}$ & - & 20,1 & 18,1 & 16,1 \\
\hline $\begin{array}{llllllllll}\operatorname{Suecia} & \ldots & \ldots & \ldots & \ldots & \ldots & \ldots & \ldots & \ldots & \ldots\end{array}$ & 67,9 & - & - & 13,8 \\
\hline $\begin{array}{llllllllll}\text { Portugal } & \ldots & \ldots & \ldots & \ldots & \ldots & \ldots & \ldots & \ldots & \ldots\end{array}$ & 90,9 & 23,8 & 21,2 & 19,6 \\
\hline 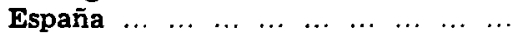 & 81,6 & 20,9 & 19,6 & 19,4 \\
\hline
\end{tabular}

FUENTE: Anuario Estadístico de los años correspondientes. 


\section{CUADRO 2}

Evolución de la tasa bruta de natalidad en España, 1900-1979

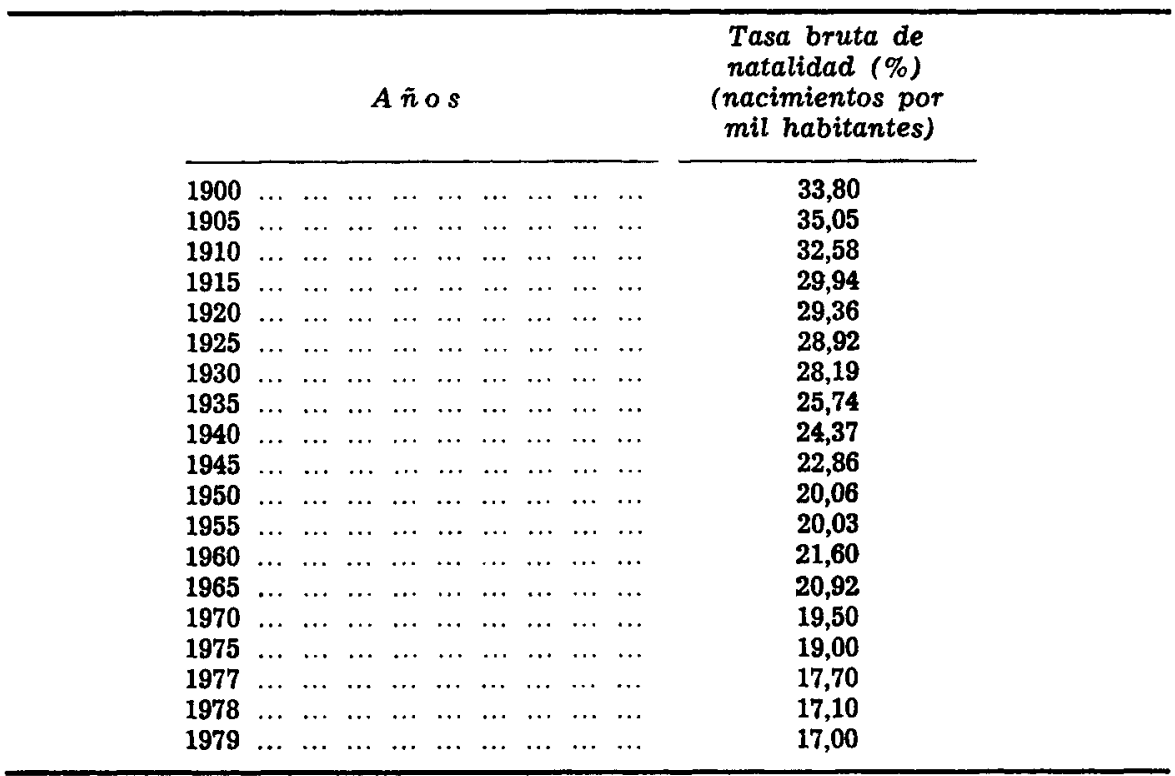

FubNTE: Anuario Estadistico de los años correspondientes.

En el cuadro 2 se observa mejor la importancia del descenso y algunas de sus características. Así, por ejemplo, podemos observar cómo únicamente existen dos períodos de incremento en las tasas brutas de natalidad: los referidos a 1900-1905 y 1955-1960. Frente a éstos, existen quinquenios con claros descensos: $1905-1910,1910-1915,1930-1935$ y $1945-1950$, que, como decíamos anteriormente, reflejan los grandes vacíos producidos tanto por las diferentes guerras como por la crisis económica o la gripe de 1918.

Así pues, el declinar de la fecundidad en España se inicia en 1905, para tomar un gran auge en el segundo cuarto de siglo, en el que se consigue un descenso de 8,86 puntos (del 28,92 al 20,06 por 100 ), y pasa posteriormente y a partir de 1960 a presentar un carácter sostenido en el descenso, aunque éste ya no sea tan espectacular (únicamente 1,06 puntos en el siguiente cuarto de siglo, desde 1950 a 1975).

El declive de la fecundidad en España responde, pues, a un cambio real en el comportamiento reproductivo de las parejas o, lo que es lo mismo, este cambio no se debe a alteraciones en las pautas de nupcialidad, ya que la tasa de nupcialidad, como podemos observar en el cuadro 3 , se mantiene en unos valores bastante sostenidos desde 1905 . 


\section{CUADRO 3}

Evolución de la tasa bruta de nupcialidad en España, 1905-1975

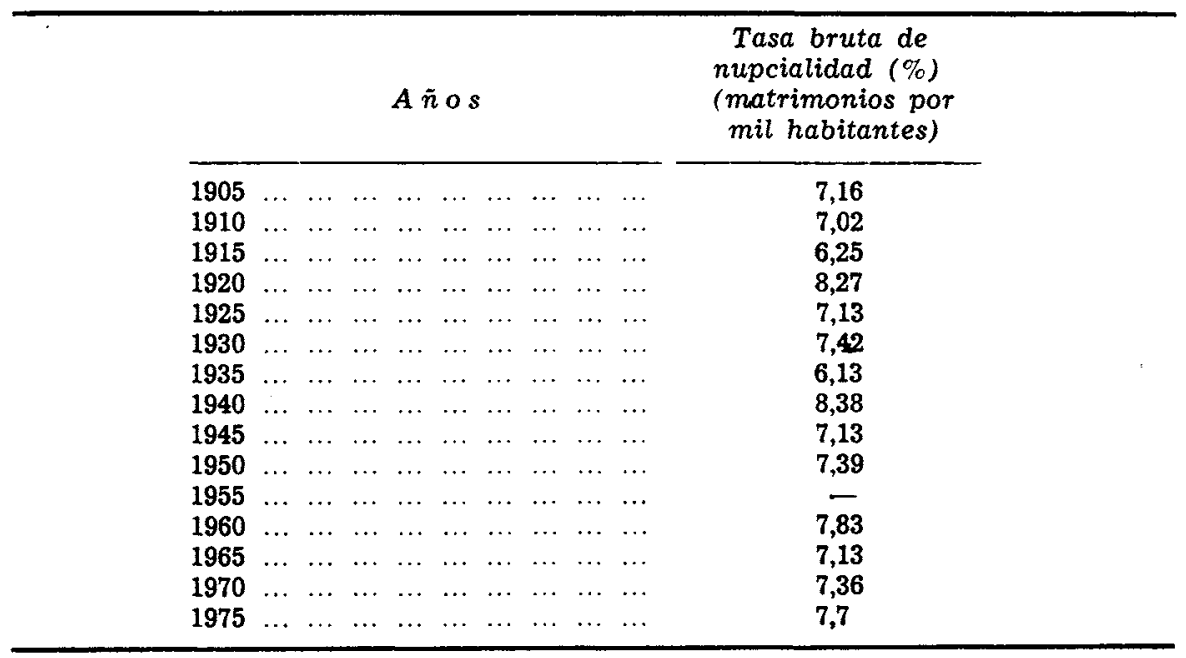

Fuenre: Anuario Estadistico de los años correspondientes.

La población española, pues, ha adoptado pautas de fecundidad controlada, reduciendo el número de hijos no por cambios significativos en los patrones de nupcialidad, sino por una paulatina generalización de la práctica anticonceptiva.

Sin embargo, se conoce poco sobre los factores que llevaron y llevan a las parejas a reducir su descendencia. Como una generalización que en sí misma no pretende explicar el fenómeno, se puede afirmar que la reducción del tamaño de la familia es un hecho que se ha presentado estrechamente vinculado al fenómeno de la modernización, ocurrido en las últimas décadas en todos los órdenes de la sociedad y de la economía española. 


\section{CRITICA DE LIBROS}

